\title{
Effectiveness and Costs of Identification Pathways for Tuberculosis: Modelling the Impact of Dual Energy X-Ray Technology
}

Karim S Karim ( $\sim$ kkarim@uwaterloo.ca )

University of Waterloo

\section{Zahid Butt}

University of Waterloo

Hamidah Hussain

Interactive Research and Development

David Bell

KA Imaging

Evan Lee

KA Imaging

\section{Susan Horton}

University of Waterloo

\section{Research Article}

Keywords: dual energy chest X-ray, computer-aided diagnostic software, cost, TB screening

Posted Date: February 25th, 2022

DOI: https://doi.org/10.21203/rs.3.rs-1386070/v1

License: (1) This work is licensed under a Creative Commons Attribution 4.0 International License.

Read Full License 


\section{Abstract \\ Background}

Existing screening methods for TB involve trade-offs among sensitivity, specificity and cost. Recent advances mean that dual-energy $\mathrm{X}$-ray is more sensitive and only slightly more costly than conventional chest X-ray and could be a high-performing alternative for TB screening, particularly if associated computer-aided diagnostic (CAD) software were developed.

\section{Methods}

Existing data on sensitivity,specificity and cost were used for six screening methods (sputum testing, conventional chest X-ray with and without CAD software, dual-energy X-ray with and without CAD software, and nucleic acid amplification testing (NAAT), used in various sequences. Cost and effectiveness of 20 different screening pathwayswere examined using data for Pakistan. Monte-carlo based sensitivity analysis focused on parameters for dual-energy X-ray.

\section{Results}

Sputum followed by NAAT is the best pathway when government budgets are low, and NAAT alone is the best when budgets are unlimited. With intermediate budgets, pathways including dual-energy X-ray dominate those including conventional $X$-rays, particularly if CAD software is developed for dual-energy X-ray.

\section{Conclusions}

Dual-energy X-ray can be a valuable addition to the screening options for TB. Our study provides different TB screening options for policy makers and TB program managers, which could be used as a guide for planning and implementation of TB screening programs.

\section{Introduction}

The past decade has seen renewed interest in halting the spread of TB through the introduction of new screening technologies [1, 2]. These either replace or complement the sputum smear test that has been the gold standard for the positive identification of tuberculosis (TB) in many countries for the past 50 years [3]. For example, molecular rapid diagnostic tests have been recommended by WHO to replace sputum smear for the confirmatory diagnosis of TB [4], reducing time-to-result and improving sensitivity and repeatability. Artificial intelligence and computer aided diagnosis (CAD) software that can perform an automated reading on chest X-ray (CXR) radiographic devices has also gained support $[5,6]$ although 
there remain challenges [7]. Another advancement is dual energy chest X-ray (DE-CXR), a higher sensitivity substitute for CXR [8-10].

Meeting the goals of WHO's End TB Strategy [11], which calls for an $80 \%$ drop in new cases of TB by 2030 will require a significant increase in overall TB case detection [12-14]. The most established rapid diagnostic test is the Xpert MTB/RIF (Xpert) was first recommended by WHO in 2010. However, the expense of the single use cartridges makes it imperative for each country to develop its own costeffective approach to TB screening, using the different available tools including CXR and DE-CXR.

DE-CXR [9], is known for higher sensitivity than CXR (at comparable specificity) in finding tuberculosis [15], lung cancer screening [16], pneumothorax [17], pneumonia [18], and coronary calcium [19]. DE-CXR obtains three X-ray images (i.e. CXR, soft tissue and bone tissue) that improves sensitivity relative to CXR. However, DE-CXR historically has not been widely used for TB detection due to higher costs, lack of portability and higher radiation [9,] although newer single exposure DE-CXR detectors are promising because they maintain CXR radiation dose and clinical technique [10, 15].

The objective of this study is to analyze the effectiveness (true and false positives including cost) of TB identification pathways that serve as a guide for national TB programs in the strategic planning process.

\section{Methods}

The tests modeled in this investigation and their respective sensitivities and specificities based on previously reported values are listed in Table 1 below. 
Table 1

Sensitivity and Specificity of various TB screening and diagnostic tests under evaluation

Test

Sensitivity Specificity

Computer Aided Diagnostic CAD4TB Software with chest X-ray with no radiologist intervention

$\begin{array}{lll}50 \text { Threshold - CAD50 [2] } & 97 & 30 \\ 80 \text { Threshold - CAD80 [2] } & 91 & 51 \\ 90 \text { Threshold - CAD90 [2] } & 85 & 66 \\ \begin{array}{l}\text { Dual Energy Chest X-ray with 50 Threshold - DE-CAD (estimated from } \\ \text { Fig. 1b) }\end{array} & 97 & 66 \\ \text { X-ray } & & \\ \text { Digital Chest X-ray read by radiologist - CXR [15] } & 70 & 81 \\ \text { Dual energy Chest X-ray read by radiologist - DE-CXR [15] } & 87 & 79 \\ \text { Diagnostic tests } & & \\ \text { Smear Microscopy - Sputum [27] } & 77 & 97 \\ \text { GeneXpert NAAT - Xpert [2] } & 98 & 96\end{array}$

Table 1 here

The first batch of tests are based on computer software using published values from CAD4TB, one of the most established CAD software for detecting TB [2]. CAD4TB uses thresholds (e.g. 50\%, 80\%, 90\%) to identify abnormality or disease and there is a well known trade-off between sensitivity and specificity such that increasing sensitivity leads to a decreasing specificity. This trade-off is less indicative of CAD software quality and more telling of the shortcomings of the chest X-ray (CXR) technique. CXR is well known for its deficiencies in differentiating soft tissue from bone for a variety of pulmonary diseases [9]. The best achievable field performance with CXR is reflected in the sensitivity and specificity numbers reported in TB studies where highly trained radiologists were involved [15]. CXR is not capable of providing a definitive diagnosis for TB without accompanying microbiological testing [2]. Thus, to maximize true positives, the highest sensitivity value for CAD4TB (i.e. the 50 threshold or CAD50) is used in our model since CAD acts as pre-screening step before confirmatory microbiological testing with sputum smear testing or a molecular rapid diagnostic test.

The sensitivity and specificity values for both CXR and DE-CXR were obtained as a weighted average of suspect TB consolidation and cavitation cases [15]. These values were chosen from the only reputable published comparison available between CXR and DE-CXR performance for TB [15]. The CXR sensitivity and specificity values used in our analysis [15] were validated by plotting the reported sensitivities of CAD4TB for three different thresholds (50,80 and 90). As shown in Fig. 1a below, the sensitivity and 
specificity values reported for CXR [15] follow a logical pathway based on reported CAD4TB data [2] based on the 50, 80 and 90 threshold of CAD4TB.

Since there is no CAD tool available today that leverages higher sensitivity DE-CXR data, a value for CAD for DE-CXR (or CAD-DE) was estimated using the relative difference between the reported sensitivities [15] of CXR and DE-CXR as shown in Fig. 1 b.

Figure $1 \mathrm{a}$ and $1 \mathrm{~b}$ here

To compare the different combination of tests, we assumed a screening population size of $1,000,000$ with a prevalence rate of 310 per 100,000 [20] based on the city of Karachi, Pakistan as a model, due to the high public health importance of screening in this population. Sequential and simultaneous testing values for sensitivity and specificity were calculated using traditional methods [21]. In Table 2 below, the different combinations of sequential and simultaneous tests evaluated are shown. The existing gold standard of sputum smear microscopy (termed "Sputum") and the newer more accurate GeneXpert NAAT (termed "Xpert") are included, as well as conventional CXR with a human reader ("CXR") and singleexposure DE-CXR with a human reader ("DE-CXR") as well as CXR with CAD ("CAD50") and DE-CXR with $C A D$ ("CAD-DE"). The combinations were chosen to reflect existing practice as well as to examine some promising alternatives. In general, we aimed to begin with a highly sensitive test and then to proceed to eliminate false positives by following up with a more specific test or tests. As per WHO recommendations, all the pathways except 20 conclude with a confirmatory microbiological test, prior to initiating treatment. 
Table 2

Combinations of screening and diagnostic tests evaluated

\begin{tabular}{|c|c|c|c|}
\hline Diagnostic pathway & First test & Second test & Third test \\
\hline 1 & CAD 50 & CXR & Sputum \\
\hline 2 & CAD 50 & CXR & Xpert \\
\hline 3 & CAD 50 & Xpert & \\
\hline 4 & Sputum & & \\
\hline 5 & CAD 50 & DE-CXR & Xpert \\
\hline 6 & Xpert & & \\
\hline $7 *$ & CAD $50+C X R$ & Xpert & \\
\hline $8^{*}$ & CAD50 + DE-CXR & Xpert & \\
\hline 9 & CAD 50 & Sputum & \\
\hline 10 & CXR & Xpert & \\
\hline 11 & DE-CXR & Xpert & \\
\hline 12 & CXR & Sputum & \\
\hline 13 & DE-CXR & Sputum & \\
\hline 14 & Xpert & Xpert & \\
\hline 15 & CAD 50 & Xpert & Xpert \\
\hline 16 & CAD-DE & Xpert & Xpert \\
\hline 17 & CAD-DE & Xpert & \\
\hline 18 & Sputum & Xpert & \\
\hline 19 & CAD-DE & Sputum & \\
\hline 20 & Sputum & CXR & \\
\hline
\end{tabular}

Table 2 here

\section{Cost estimation}

The direct costs in USD from the healthcare payer perspective for the tests and first round of treatment are shown in Table 3 [22]. These costs were verified using the lowest single unit price obtained in August 2021 (converted using the exchange rate as of August 31, 2021 [23]) from one of three testing 
laboratories (Aga Khan University Hospital, Essa Laboratory, Ziauddin Hospital) in Karachi, Pakistan. The quoted price at these laboratories was slightly higher compared to the values reported in literature [22] potentially because the testing laboratories were private and not dedicated TB facilities. For newer tests like DE-CXR, the baseline cost was estimated as being $25 \%$ higher than CXR [24]. All costs were estimated for a single round of screening and treatment within a single year; hence no discounting of costs was applied.

Table 3

Costs per test and treatment

\begin{tabular}{|c|c|c|}
\hline Screening or Diagnostic Test & $\begin{array}{l}\text { Cost (converted to } \\
\text { USD) }\end{array}$ & Reference \\
\hline Chest X-ray with no reader & $\$ 1.70$ & 22 \\
\hline Dual energy Chest X-ray with no reader (estimated*) & $\$ 2.13$ & 24 \\
\hline Chest X-ray plus Human Reader (CXR) & $\$ 2.15$ & 22 \\
\hline $\begin{array}{l}\text { Dual energy Chest X-ray plus Human reader (estimated*) (DE- } \\
\text { CXR) }\end{array}$ & $\$ 2.69$ & 24 \\
\hline Chest X-ray with CAD reader (CAD50) & $\$ 2.70$ & 22 \\
\hline $\begin{array}{l}\text { Dual energy Chest X-ray with CAD reader (estimated*) (CAD- } \\
\text { DE) }\end{array}$ & $\$ 3.13$ & 24 \\
\hline Sputum Smear Microscopy (Sputum) & $\$ 1.26$ & 22 \\
\hline GeneXpert NAAT (Xpert) & $\$ 21.28$ & 22 \\
\hline TB Treatment & $\$ 114.82$ & 22 \\
\hline \multicolumn{3}{|c|}{$\begin{array}{l}\text { *The assumption for CAD-DE is that the cost of conducting the X-ray is } 25 \% \text { more than for } \\
\text { conventional X-ray, and the cost of a human reader for CAD-DE is similarly } 25 \% \text { more than for } \\
\text { conventional X-ray, but there is no difference in the cost of an Al reader }(\$ 1.00) \text { for CXR and DE-CXR. }\end{array}$} \\
\hline
\end{tabular}

Table 3 here

\section{Sensitivity Analysis}

Since costs for DE-CXR are estimated, a Monte Carlo sensitivity analysis was conducted using the Oracle Crystal Ball extension for Microsoft Excel with 10,000 trials. The per image cost of DE-CXR equipment was modeled as a normally distributed random variable with a mean cost of $\$ 1.87$ (i.e. $10 \%$ greater than CXR) and a standard deviation of 5 cents. The specificity of DE-CXR was also modeled as a normally distributed random variable with a mean specificity of $51 \%$ (about 20 absolute percentage points higher than CAD50 for the same sensitivity of $97 \%$ with a standard deviation of $9 \%$. The standard deviation was chosen so that the specificity could drop a little below CAD50 (i.e. $30 \%$ ) in the worst and reach $80 \%$ (i.e. DE-CXR human reader) in the best tail case. We believe this is a reasonable assumption since CXR is a 
sub-set of DE-CXR as DE-CXR provides both a regular CXR plus DE images. The CHEERS checklist for economic evaluations was followed throughout [25].

\section{Statistical Analysis}

In our analysis, the goal is to maximize the number of true cases treated, at a given cost. The size of the government budget would then determine how many cases can be identified and treated, such that the testing modality chosen may change as government budgets for TB screening and treatment increase. This favours pathways with lower screening costs, and fewer false positives who are treated, for a given number of true positive patients who are treated. Note also that, in our first order analysis, we do not explicitly consider non-cost impacts on the health system, such as the impact of tests such as Xpert that are more labour-intensive or have lower throughputs in contrast to CXR.

\section{Results}

In Fig. 2, for each of the testing pathways, results in terms of true cases found (true positives) and true cases missed (false negatives) are summarized. If we use Sputum Smear Testing (Pathway 4) as the gold standard, any pathway that cannot achieve a similar sensitivity value (i.e. $77 \%$ ) will face some resistance in adoption. Pathway 4 then rules out a number of alternate pathways (e.g. pathways 1, 2, $9,10,10,12,13$ and 20 ) which are no less costly but are less sensitive than sputum alone. This result also rules out some prominent pathways. i.e. since if CXR with a human reader yields a sensitivity of $70 \%$ (see Table 1), and hence two low sensitivity tests (ie. CXR and Sputum) taken in series yields a sensitivity of around $50 \%$ (see Pathway 12 and 20 ).

Figure 3 shows the number of true cases (true positives) and false positives for each of the testing pathways, or alternately, how many persons are being unnecessarily treated for TB. For example, the gold standard Sputum (Pathway 4) detects ten times as many false positives as true positives because its specificity is $97 \%$, implying that $3 \%$ of the screened population (or nearly 30,000 out of the $1,000,000$ screened) would be incorrectly identified as TB positive.

Figure 3 here

The high number of false positives has ramifications due to increased program and patient costs, increased antibiotic resistance, and quality of life concerns for those being unnecessarily treated. In order to minimize false positives, several prominent pathways require scrutiny. For example, the gold standard Pathway 4 (Sputum only) and Pathway 6 (Xpert only) both result in a very large number of false positives. One method to suppress false positives (albeit at increased cost) is to double up on a high sensitivity, high specificity microbiological test e.g. performing two Xpert tests in series. In this case, pathways $14,15,16$, and 18 become attractive.

Figure 4a plots the true positives found versus the detection and treatment costs of each test normalized to the most expensive pathway (Pathway 6: Xpert only) in Table 2. It comes as no surprise that here, Pathway 6 (Xpert only) is the most expensive due to the up to ten times higher cost relative to Sputum, in 
addition to the additional costs of false positives requiring treatment. The lower envelope of the points provides the expansion pathway. The inclusion of DE-CXR as an option means that the expansion path is pathway 18 (Sputum/Xpert), pathway 17 (CAD-DE/Xpert), pathway 8 (CAD-50 in parallel with DE-CXR) and finally pathway 6 (Xpert only). If DE-CXR is not an option, the expansion path is instead pathway 18 (sputum/Xpert, pathway 7 ( 2 simultaneous CXR, one read by a radiologist and one read by CAD), and finally pathway 6 (Xpert only). The expansion path with CXR rather than the DE-CXR option is inferior (costing more to treat the same number of true positives).

Figure 4 here

CAD software coupled with conventional CXR, in general, is promising only if it is coupled with a high sensitivity, high specificity follow-on test like Xpert to confirm true positivity (e.g. pathway 7). For today's CAD solutions [26] that use CXR, the normalized cost is significantly more than Sputum-only testing, with cost minimization limited by both the sensitivity-specificity trade-off shown in Fig. 1a and the high cost of Xpert cartridges.

It is challenging for CAD using CXR data only to achieve a breakthrough in TB screening efficiency since the upper detection limit is set by the physics of conventional X-ray imaging which is poor at distinguishing soft tissue overlaid by bone, even when read by expert human readers [15-19]. However, dual energy X-ray offers an intriguing alternative by providing substantially higher sensitivity without compromising specificity. A CAD or Al software developed using DE-CXR data (termed CAD-DE as noted previously) could help realize a high true positive and low false positive combination ideal for mass automated screening of TB using existing chest X-ray infrastructure.

The Monte Carlo sensitivity analysis is shown in Fig. 4b where Pwy 17 (CAD-DE followed by Xpert) is compared to Pwy 3 (CAD50 followed by Xpert). In almost all cases (98.8\%), Pwy 17 yields a superior economic outcome compared to Pwy 3 and it is evident that even in pessimistic scenarios, DE-CXR continues to dominate CXR.

\section{Discussion}

Strengths of this study include examination of a novel X-ray technology that can provide a middle alternative to a low cost option which misses many cases (Sputum) and a high cost option which involves treatment of a significant number of false positives (Xpert). Limitations involve the assumptions made on sensitivity and specificity of the CAD-DE combination, in the absence of an already-developed software.

The results illustrate the trade-offs between the detection pathways and screening and treatment costs where false positives lead to unnecessary treatment costs and false negatives lead to the perpetuation of infection. None of the existing tests are perfect. That leads to consideration of sequences of tests using a highly sensitive test at the outset to capture the highest number of true positives followed by a test with 
high specificity to eliminate false positives (that drive up treatment costs and causing disutility to those treated unnecessarily, and potentially worsening antimicrobial resistance).

Sequential tests do, however face potential issues with loss to follow up. These can be minimized if it is possible to take multiple sputum samples from the same individual for a confirmatory test, only if the initial test indicates this is appropriate, in pathways that employ both a sputum test and Xpert, or two Xpert tests in sequence. There are two issues here: firstly, some observers have suggested that it is difficult enough for some patients to produce one sputum sample, let alone two (if both tests require sputum), and secondly, it requires technology to preserve the second sputum sample until analysis of the first sample is complete.

Our analysis suggests that pathways involving Sputum followed by Xpert (Pathway 18), DE-CXR followed by Xpert (Pathway 11), and CAD-DE followed by Xpert (Pathway 17), may dominate other pathways by offering a high-sensitivity initial test at relatively low cost to maximize true positives found, followed by a more expensive test with higher specificity to reduce over-treatment of false positive cases. If DE-CXR becomes widely adopted, Pathway 17 becomes the path giving significantly better outcomes at costs that are not very different from today's Sputum only gold standard.

The above discussion is predicated on expediting the successful development of a CAD/AI software that leverages the additional data from DE-CXR as well as the adoption of DE-CXR as a substitute for CXR. Even in the interim before a CAD-DE software is developed, there are advantages to adopting DE-CXR with human readers as Pathway 11 shows.

The high expected performance and relatively low cost of a CAD applied to DE-CXR, followed by confirmatory testing with GeneXpert lends itself strongly to investment in the development of a CAD applied to the DE-CXR images. Expected savings will rapidly cover costs of such investments.

\section{Conclusions}

This study highlights the importance of comparing different screening options for TB that can be used by policymakers for decision making around adopting the most cost effective and feasible screening option in their national TB control programs.

\section{Declarations}

\section{Ethics}

This modelling study used public data and did not require ethical approval

\section{Consent to publish}

Not applicable 
Availability of data and materials

All data used for the model are included in the article. Calculation spreadsheets are available from the corresponding author upon reasonable request.

\section{Competing interests}

KSK is Chief Technology Officer and DB and EL are consultants for KA Imaging, which markets dualenergy X-ray systems. $\mathrm{SH}, \mathrm{HH}$ and ZB declare no competing interests.

\section{Funding}

No funding was received for this study

\section{Author contributions}

KSK conceptualized the study and analysis design. AB, DB, EB, ZB, SH contributed to the analysis design. $\mathrm{KSK}, \mathrm{SH}$ undertook the calculations. KSK wrote the first draft of the manuscript. All authors reviewed and approved the final manuscript.

\section{Acknowledgements}

KSK thanks Sahar Jessa and Aisha Siddiqui for discussions and pricing information on TB related services in Pakistan.

\section{References}

1. Blakemore R, Story E, Helb D, Kop J, Banada P, Owens MR, et al. Evaluation of the analytical performance of the Xpert MTB/RIF assay. J Clin Microbiol. 2010;48:2495-2501.

2. Zaidi, SMA, Habib SS, Van Ginneken B, Ferrand RA, Creswell J, Khowaja S, et al. Evaluation of the diagnostic accuracy of Computer-Aided Detection of tuberculosis on chest radiography among private sector patients in Pakistan. Sci Rep. 2018;8:1-9.

3. Yablonskii PK, Vizel AA, Galkin VB, Shulgina MV. Tuberculosis in Russia. Its history and its status today. Am J Respir Crit Care Med. 2015:191:372-376.

4. World Health Organization. Xpert MTB/RIF implementation manual: technical and operational 'howto'; practical considerations. No. WHO/HTM/TB/2014.1. World Health Organization, 2014.

5. Qin ZZ, Ahmed S, Sarker MSS, Paul K, Adel ASS, Naheyan T, et al. Tuberculosis detection from chest $x$-rays for triaging in a high tuberculosis-burden setting: an evaluation of five artificial intelligence algorithms. Lancet Digit Health. 2021;3:e543-e554. https://doi.org/10.1016/S2589-7500(21)00116-3

6. World Health Organization. Ethics and governance of artificial intelligence for health: WHO guidance. Geneva: WHO, 2021. https://www.who.int/publications/i/item/9789240029200 
7. Roberts M, Driggs D, Thorpe M, Gilbey J, Yeung M, Ursprung S, et al. Common pitfalls and recommendations for using machine learning to detect and prognosticate for COVID-19 using chest radiographs and CT scans. Nat Mach Intell. 2021;3:199-217.

8. Mogami H, Onoike Y, Miyano H,Arakawa $K$, Inoue H, Sakae $K$, et al. Lung cancer screening by singleshot dual-energy subtraction using flat-panel detector. Jpn J Radiol. 2021: June 26. Doi:

10.1007/s11604-021-01163-z

9. Karim, KS. Single-Exposure, Digital, Dual-Energy Subtraction X-Ray Ushers in a New Era of Diagnostic X-Ray Imaging. Radiol Manage. 2021; Mar/Apr: 23-28.

10. Maurino S, Lopez S, Ghanbarzadeh S, Karim K. Novel multi-energy x-ray detector allows for simultaneous single-shot acquisition of digital radiography and tissue-subtracted images. European Congress of Radiology-ECR 2019, 2019. https://epos.myesr.org/poster/esr/ecr2019/C-3350 (Accessed November 18, 2021).

11. World Health Organization. The End TB Strategy. Available from: https://www.who.int/teams/globaltuberculosis-programme/the-end-tb-strategy (accessed November 8, 2021).

12. World Health Organization. WHO consolidated guidelines on tuberculosis. Module 2: screening systematic screening for tuberculosis disease. Geneva: World Health Organization, 2021. Licence: CC BY-NC-SA 3.0 IGO. https://apps.who.int/iris/bitstream/handle/10665/340255/9789240022676eng.pdf (accessed November 8, 2021).

13. World Health Organization. WHO consolidated guidelines on tuberculosis. Module 3: Diagnosis Rapid diagnostics for tuberculosis detection 2021 update. https://www.who.int/publications/i/item/9789240029415 (Accessed October 5, 2021).

14. Cilloni L, Fu H, Vesga JF, et al. The potential impact of the COVID-19 pandemic on the tuberculosis epidemic: a modelling analysis. EclinicalMedicine. 2020;28:100603.

15. Sharma M, Sandhu MSS, Gorsi U, Gupta D, Khandelwal N. Role of digital tomosynthesis and dual energy subtraction digital radiography in detection of parenchymal lesions in active pulmonary tuberculosis. Eur J Radiol. 2015;84:1820-1827.

16. Mogami H, Onoike Y, Miyano H, Arakawa K, Inoue H, Sakae K et al. Lung cancer screening by single-shot dual-energy subtraction using flat-panel detector. Jpn J Radiol. 2021;39:1168-1173

17. Urbaneja A, Dodin G, Hossu G, Bakour O, Kechidi R, Teixeira PG, et al. Added value of bone subtraction in dual-energy digital radiography in the detection of pneumothorax: impact of reader expertise and medical specialty. Acad Radiol. 2018;25:82-87.

18. Martini K, Baessler M, Baumueller S, Frauenfelder T. Diagnostic accuracy and added value of dualenergy subtraction radiography compared to standard conventional radiography using computed tomography as standard of reference. PloS One. 2017;12:e0174285.

19. Song Y, Wu H, Wen D, Zhu B, Graner P, Ciancibello L, et al. Detection of coronary calcifications with dual energy chest X-rays: clinical evaluation. Int J Cardiovasc Imaging. 2021;37:767-774.

20. Metzger P, Baloch NA, Kazi GN, Bile KM. Tuberculosis control in Pakistan: reviewing a decade of success and challenges. East Mediterr Health J. 2010:16 (Supp):47-53. 
21. Sergeant E, Perkins N. 2016. Epidemiology for Field Veterinarians: an Introduction. CABI: Wallingford, UK, 2016. Pp 98-102.

22. Nsengiyumva NP, Hussain $H$, Oxlade $O$, Majidulla A, Nazish A, Khan AJ, et al. Triage of persons with tuberculosis symptoms using artificial intelligence-based chest X-ray interpretation: A costeffectiveness analysis. Open Forum Infect Dis. Accepted October 2021, in press.

23. Oanda Currency Converter, https://www1.oanda.com/currency/converter/ (website accessed Aug 31, 2021).

24. Personal communication with KA Imaging, November 2021.

25. EQUATOR Network. CHEERS checklist. Items to include when reporting economic evaluations of health interventions. https://www.equator-network.org/wp-content/uploads/2013/04/RevisedCHEERS-Checklist-Oct13.pdf (accessed December 11, 2021)

26. Qure.ai. qXR Becomes First Al-Based Chest X-ray Interpretation Tool to Receive CE Certification. News item. https://qure.ai/news/2018/05/31/qXR-CE.html (accessed November 8, 2021).

27. Chadha VK Anjinappa SM, Rade K, Arakawa K, Inoue H, Sakae K, et al. Sensitivity and specificity of screening tools and smear microscopy in active tuberculosis case finding. Indian $\mathrm{J}$ Tuberc. 2019:66:99-104.

\section{Figures}


Chest X-ray/CAD sensitivity and specificity
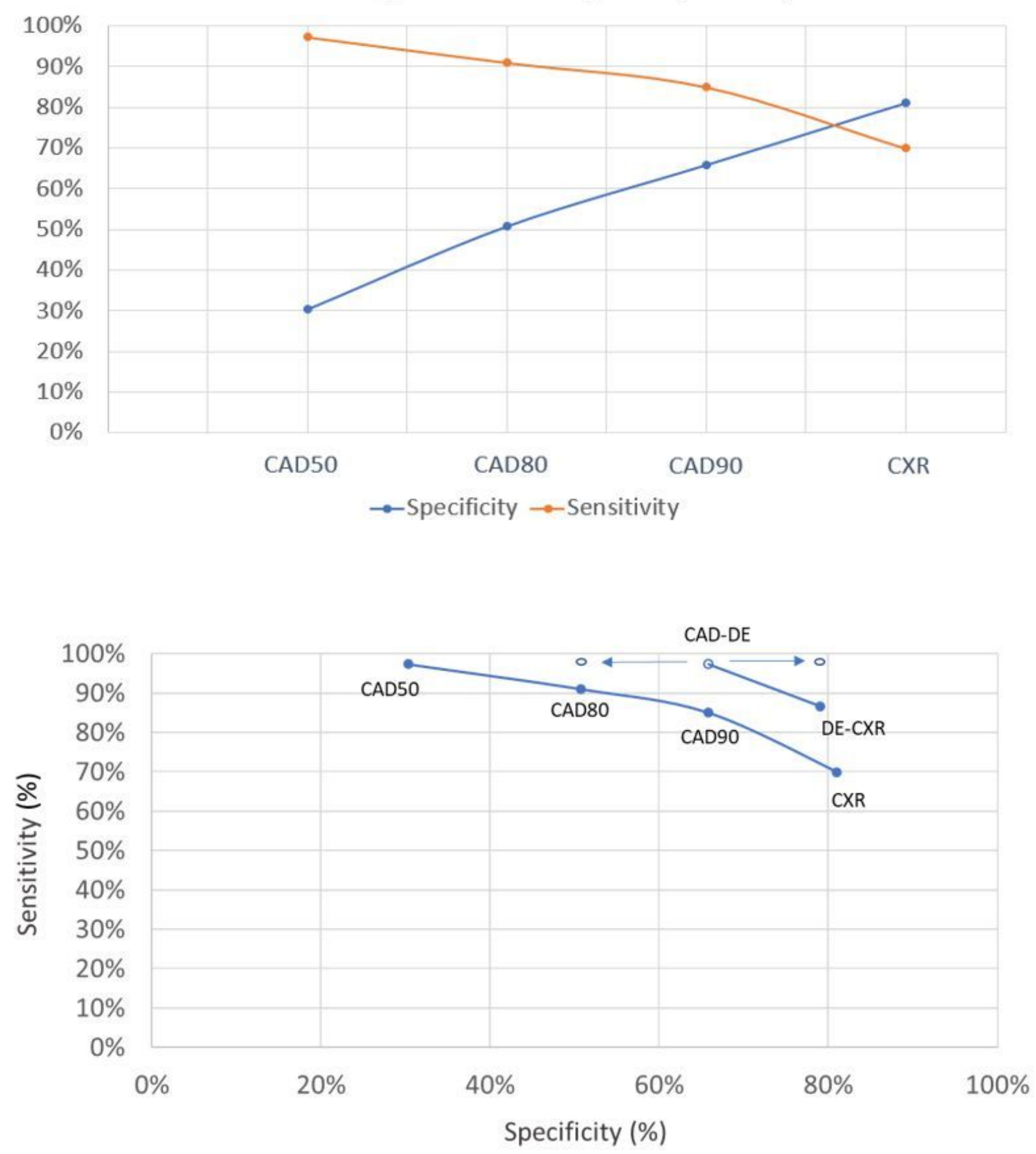

Figure 1

(a) Chest X-ray CAD sensitivity and specificity trend and (b) estimating the performance of an equivalent CAD-DE tool that uses dual energy chest $X$-ray data 
True positives (lines) and False negatives (dots) in (\%)

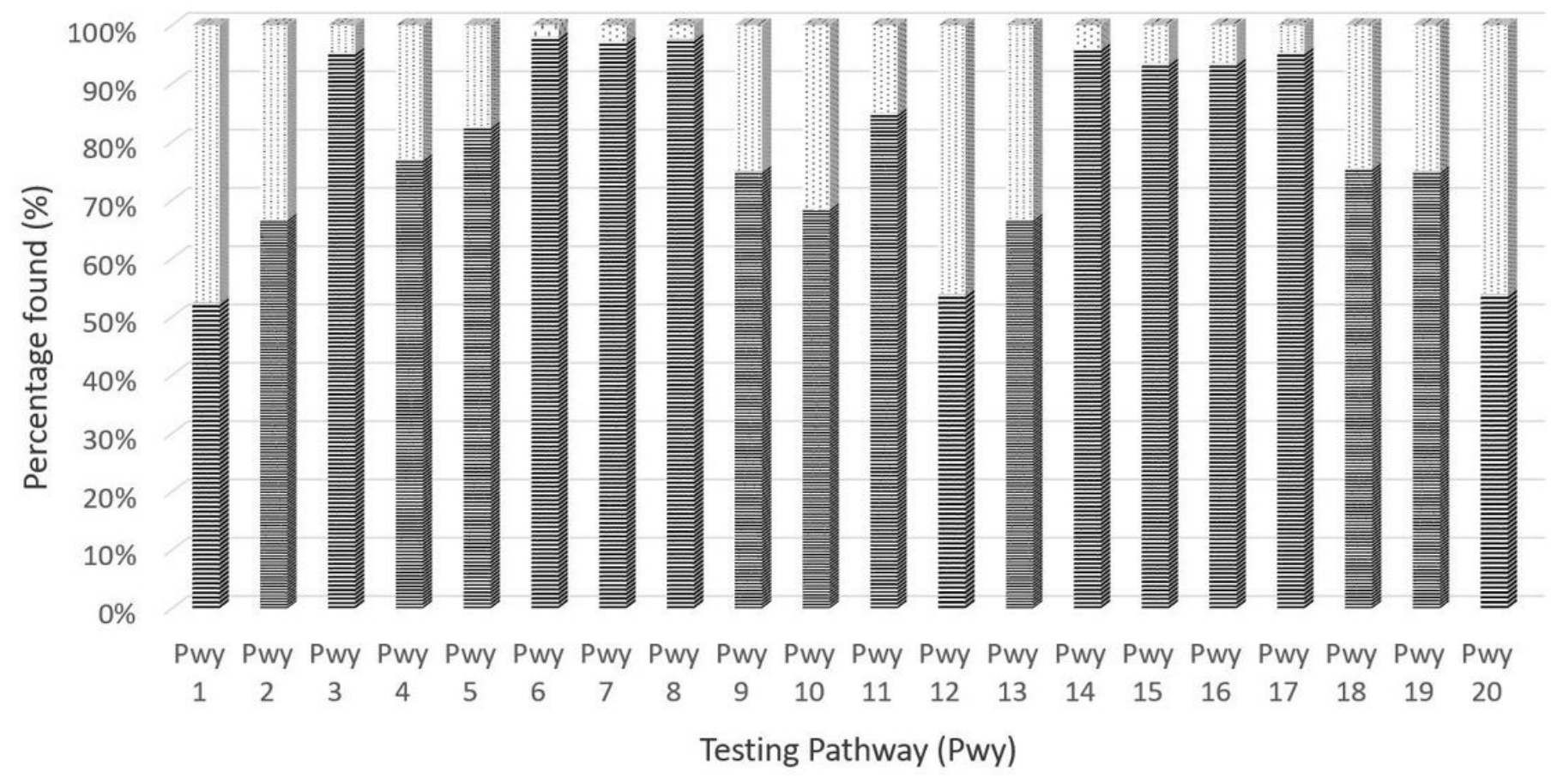

Figure 2

TB cases found and missed (\%)

Legend: True positives (lines) and false negatives (dots) in \%

True positives (lines) and False positives (crossed lines)

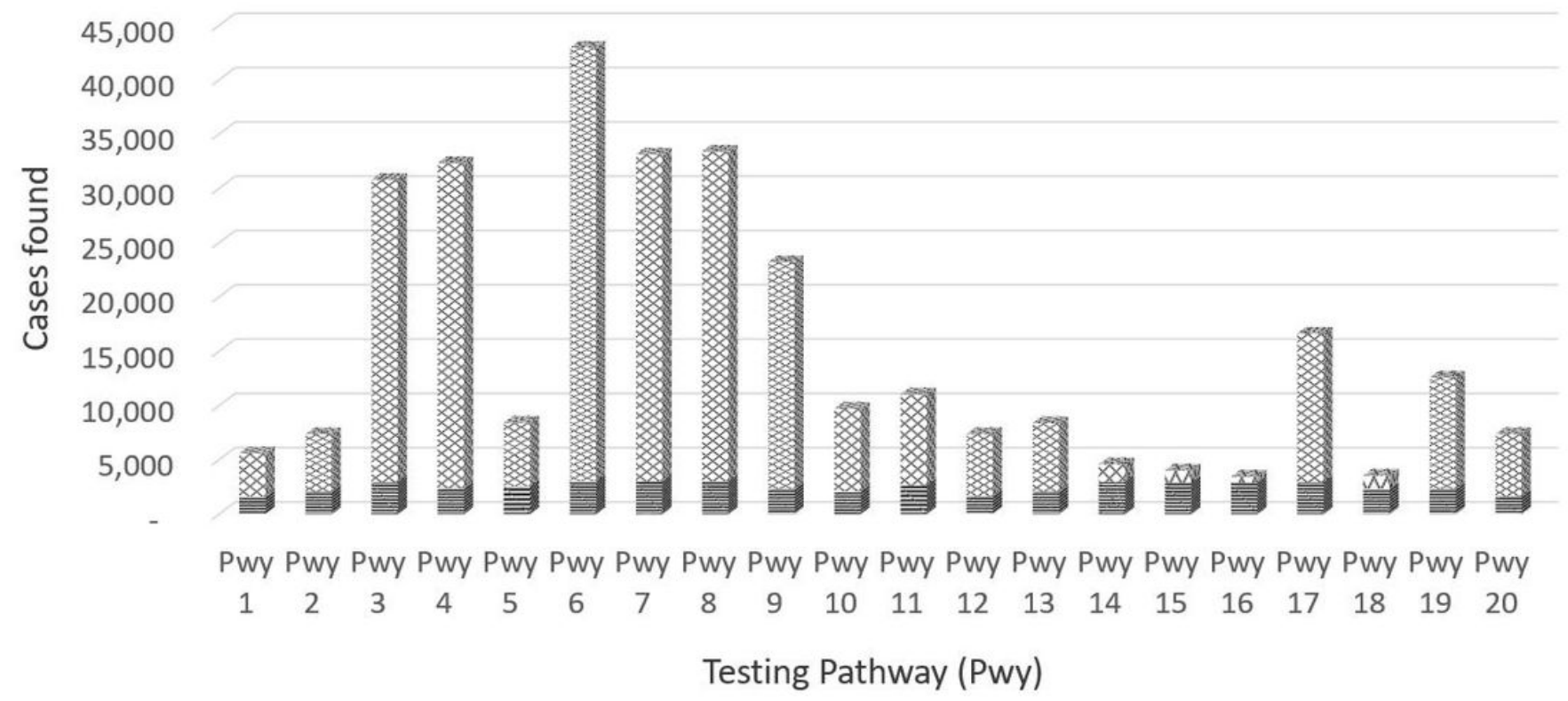




\section{Figure 3}

TB cases found correctly (true positive) and incorrectly (false positive)

Legend: True positives (horizontal lines) and false positives (cross-hatching)
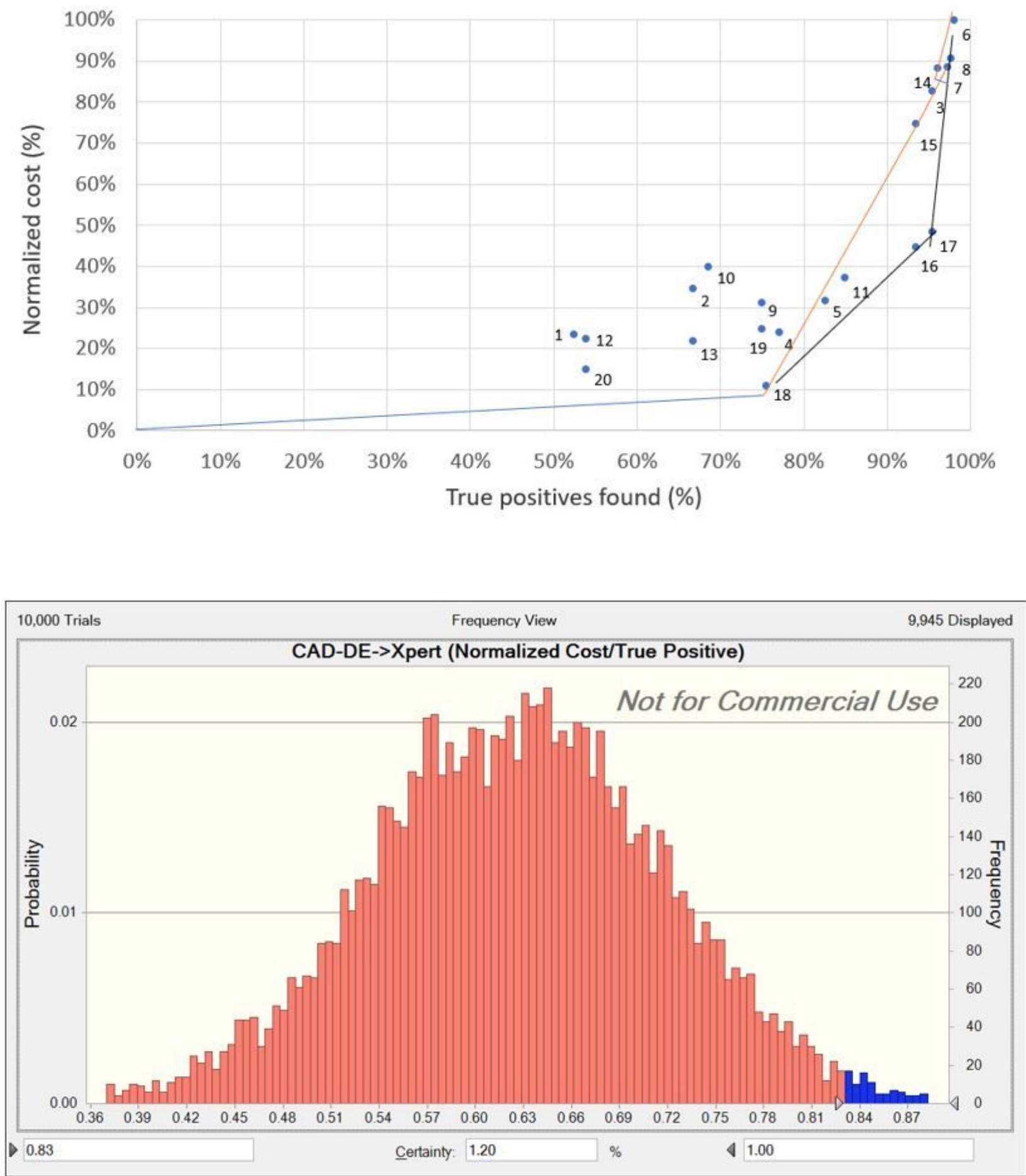

Figure 4 
(a) Expansion path comparing normalized cost to true positives found and treated

Legend:

Orange: expansion path feasible with conventional X-ray (CXR) and related technologies

Black: expansion path feasible with dual energy chest X-ray (DE-CXR) and related technologies

Blue: expansion path feasible with both CXR and DE-CXR and related technologies

(b) Monte Carlo Sensitivity analysis varying both, the cost and specificity of DE-CXR relative to conventional CXR 